\title{
Chemotherapy-Induced Leukoencephalopathy: A Case Series
}

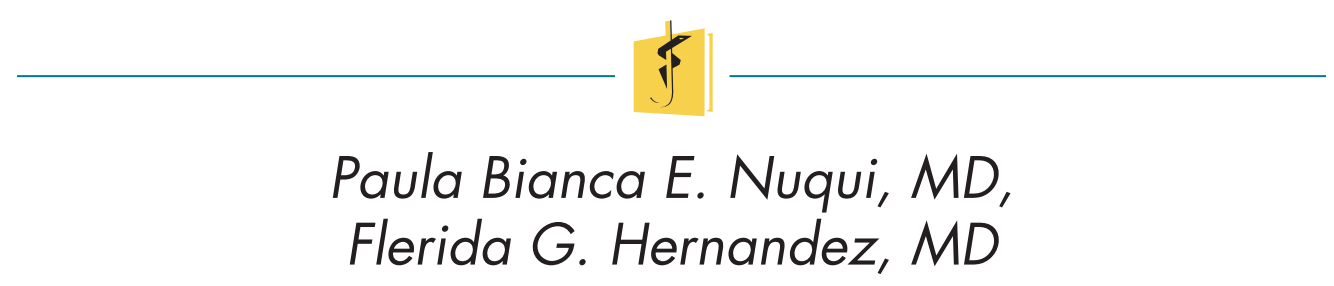

\section{ABSTRACT}

Rationale: Leukoencephalopathy, a complication associated with chemotherapy has been reported after giving high doses of methotrexate and cytarabine with no specific risk factors to date.

\section{Objectives:}

1. To review the prevalence of chemotherapy-induced leukoencephalopathy in children with acute lymphoblastic leukemia (ALL).

2. To present the clinical course, pathogenesis and neuro-imaging findings of chemotherapy-induced leukoencephalopathy in children with ALL.

Case: We reported three cases of adolescent ALL precursor B-cell patients who received high doses of methotrexate and presented with neurologic and MRI findings consistent with leukoencephalopathy. Our patients were only placed on supportive measures with adequate hydration, without providing any special intervention. Yet, all of them had complete neurological recovery.

Discussion and Summary: Methotrexate is a cell cycle-specific agent that inhibits the enzyme dihydrofolate reductase, preventing the conversion of folic acid to tetrahydrofolic acid and inhibiting cell replication. It is one of the most commonly

Paula Bianca Nuqui

paulanuqui14@gmail.com

Department of Pediatrics, Section of Hematology/

Oncology, University of Santo Tomas

Hospital, Manila, Philippines implicated drug causing leukoencephalopathy. [3] On MRI T2-weighted images, all of them had hyperintensities on the posterior frontal/parietal corona radiata and centrum semiovale consistent with leukoencephalopathy. Complete recovery happened spontaneously in all of the cases. There is no standard treatment for acute and subacute toxicities from methotrexate.

Keywords: Leukoencephalopathy, Chemotherapeutic drugs, Neurotoxicity, Case series

\section{INTRODUCTION}

Leukoencephalopathy (LEP) is a structural alteration of the cerebral white matter in which the myelin suffers most of the damage.[1] It is a complication associated with both radiation and chemotherapy and characterized clinically by dementia, ataxia and focal motor deficits, which can progress to coma and death.[2] Neurotoxicity is a common adverse effect of cancer therapeutics that often represents a dose-limiting side effect. We reviewed three cases of chemotherapy-induced LEP to describe their course, identify risk factors and pertinent clinical findings which resulted in complete resolution of symptoms in all cases. 


\section{CASE SUMMARY}

\section{Case 1}

A 10-year-old male presented with intermittent fever, cough, blood-tinged nasal discharge, oral mucosal petechiae and gum bleeding. He was pale with multiple cervical lymphadenopathies, splenomegaly, cutaneous petechial lesions and undescended left testes. Complete blood count (CBC) showed hyperleukocytosis of $102,000 / \mathrm{mm} 3$ and thrombocytopenia. He was diagnosed with acute lymphoblastic leukemia (ALL), precursor-B-cell and was given chemotherapy with modified BFM95/ HK97 ALL protocol. He developed sudden onset of left-sided weakness at 28 weeks into chemotherapy, seven days following triple intrathecal (IT) chemotherapy. The total cumulative IT methotrexate (MTX) and cytarabine doses were $132 \mathrm{mg}$ and 300 $\mathrm{mg}$, respectively.

\section{Case 2}

A 15-year-old male presented with fever, chills, loss of appetite and weakness. CBC showed leukopenia, which later progressed to pancytopenia. Bone marrow aspiration done twice showed normal results. He was initially treated for immune thrombocytopenia by giving prednisone $60 \mathrm{mg}$ per day. He subsequently developed multiple cervical lymphadenopathies and maculopapular rash on the cheeks with no splenomegaly. Prednisone was tapered and he was followed up closely. Seven months from initial symptoms, his blood count showed lymphocytosis of $96 \%$. Bone marrow flow cytometry and core biopsy revealed ALL, precursor B-cell. His white blood cell count subsequently went up to $56,000 / \mathrm{mm} 3$. He underwent chemotherapy with BFM95/HK97 ALL protocol. He developed sudden onset of left-sided jerky movements followed by left-sided weakness at 28 weeks into chemotherapy, nine days following triple IT chemotherapy. The total cumulative IT MTX and cytarabine doses were $132 \mathrm{mg}$ and $300 \mathrm{mg}$, respectively.

\section{Case 3}

A 15-year-old male presented with a week-long fever and occasional cough. No other symptoms were noted. He was treated as a case of enteric fever. $C B C$ on the third hospital day revealed pancytopenia. He was pale then with multiple cervical lymphadenopathies, petechiae and splenomegaly. $\mathrm{He}$ was diagnosed with ALL, precursor-B cell and was treated with BFM95/HK97 ALL protocol. He developed sudden onset of left-sided weakness at 14 weeks into chemotherapy, nine days following triple IT chemotherapy. The cumulative IT MTX and cytarabine doses were $84 \mathrm{mg}$ and $210 \mathrm{mg}$, respectively.

\section{Clinical Course}

All patients were classified under intermediate risk, all being at or above 10 years of age while two of them presented with initial WBC count $>50,000 /$ mm3. They all underwent BFM95/HK97 protocol, with drug doses based on ideal body weight.

\section{ALL BFM95/HK97 Protocol}

\begin{tabular}{|c|c|c|c|c|c|}
\hline$I_{A}$ & $I_{8}$ & $M$ & $I_{A}$ & $\mathrm{U}_{\mathrm{B}}$ & MT \\
\hline 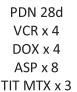 & $\begin{array}{l}\text { 6-MP 28d } \\
\text { CPM } \times 2 \\
\text { ARA-C C } \times 4 \\
\text { TIT MTX } \times 2\end{array}$ & $\begin{array}{c}\text { 6-MP 56d } \\
\text { HD-5gMTX } \times 4 \\
\text { TIT MTX } \times 4\end{array}$ & $\begin{array}{l}\text { DXM 21d } \\
\text { VCR } \times 4 \\
\text { DO } \times 4 \\
\text { ASP } \times 4\end{array}$ & $\begin{array}{l}\text { 6-MP } 14 d \\
\text { CPM } \times 1 \\
\text { ARA-C } 2 \times 4 \\
\text { TIT MTX } \times 2\end{array}$ & 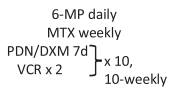 \\
\hline
\end{tabular}

Bone marrow minimal residual disease (MRD) at day 28 was negative in all three patients. Cumulative IT MTX and cytarabine were both accounted. The first two patients completed the four bi-weekly MTX $5 \mathrm{~g} /$ $\mathrm{m} 2$ and were on the last week of re-induction Phase Ilb, while the third had just received the second MTX $5 \mathrm{~g} / \mathrm{m} 2$ of protocol $M$ when they developed LEP. LEP happened seven to nine days after triple IT with hydrocortisone, MTX and cytarabine.

At presentation, all of them were conscious and coherent with stable vital signs. They were all overweight. All of them had intact mentation, central facial palsy on the left, hemiparesis on the left and Babinski on the left; while the first two had sensory deficit to light touch and pain on the left upper and left lower extremities. All of them had normal serum electrolytes. Cranial CT scans were all normal. Cranial MRI revealed signs of LEP as described in section IV. During their hospital stay, they all developed progressive weakness. The first patient eventually had bilateral weakness and decreasing sensorium; the second patient subsequently developed aphasia, drooling of saliva with progression of left-sided weakness, and the third patient had progressive weakness on the left from $4 / 5$ to $3 / 5$. All of them had crying episodes and affective disturbances. These patients were all 
managed with adequate hydration at maintenance and all had complete resolution of symptoms within 24-48 hours. They were all discharged in stable condition without deficits.

\section{IMAGING}
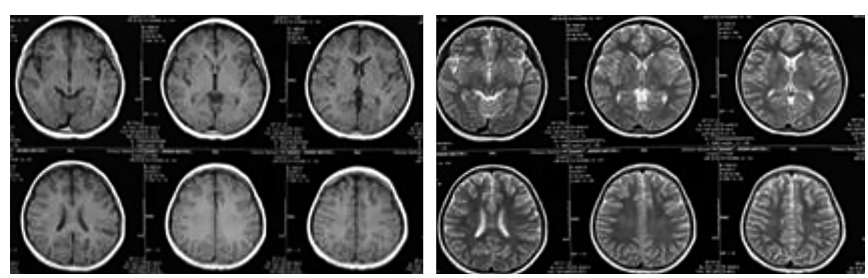

Figure 1

Case 1: T1W and T2W images

T1W: Subtle hypointensities in both frontal corona radiata and centrum semiovale

T2W: Subtle hyperintensities in both frontal corona radiata and centrum semiovale
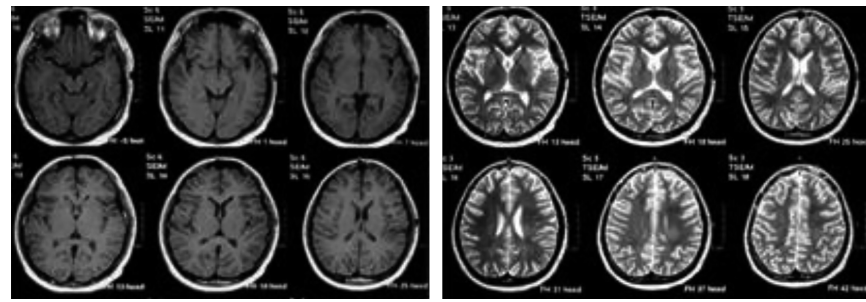

Figure 2

Case 2: T1 and T2 Weighted Images

TIW: Hypointensities on left posterior frontal/ parietal corona radiata and centrum semiovale

T2W: Hyperintensities on left posterior frontal/ parietal corona radiata and centrum semiovale white matter
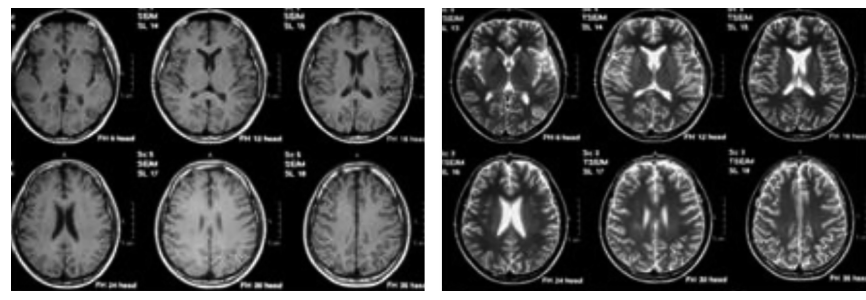

Figure 3 :

Case 3: T1 and T2 Weighted Images

T1W: Hypointensities on left parietal parasagittal area

T2W: Subtle hyperintensities on left parietal parasagittal area

\section{DISCUSSION}

Neurotoxicity is a common side effect of chemotherapeutic drugs. It may lead to disability and decreased quality of life. The most common manifestations include peripheral neuropathy and encephalopathy. Often, symptoms resolve or improve upon removal of the offending agent.[3]

\section{Incidence of LEP}

Filley et al. in 2010 explained that neurotoxicity depends on the route of delivery, the dose and the drug used. MTX-induced LEP may occur in less than $10 \%$ of patients receiving intravenous MTX and $40 \%$ of those receiving IT MTX.[4] Ziereisen in 2006 reported that $16.7 \%$ (15) of the 90 patients who were given high-dose MTX at $5 \mathrm{~g} / \mathrm{m} 2$ showed MRI evidence of LEP, nine of whom were asymptomatic and six were symptomatic.[5] MRI normalization occurred later than clinical recovery in these six patients.

Baker et al. in 1991 reported that $3-26 \%$ of patients given cytarabine at a dose more than $1 \mathrm{~g} /$ $\mathrm{m} 2$ or higher presented with a series of neurological side effects such as cerebellar dysfunction, gait disturbances, seizures or death. Approximately only $40 \%$ of the patients recovered.[6] From the Critical Reviews in Oncology/Hematology, cerebellar dysfunction particularly occurred in older patients at a cumulative dose of $36 \mathrm{~g} / \mathrm{m} 2$ but symptoms have been described to come out at a dose as low as 3 $\mathrm{g} / \mathrm{m} 2$; while in the pediatric population symptoms tend to occur when cytarabine was given via the IT route.[7]

\section{Presentation}

MTX neurotoxicity may present with aseptic meningitis, myelopathy, stroke-like focal deficits, seizures or chronic encephalopathy. Cytarabine neurotoxicity may present with encephalopathy, seizures, cerebellar dysfunction, aseptic meningitis and myelopathy. [8] The resolution of symptoms varies between these two chemotherapeutic drugs. MTX LEP results in complete resolution of symptoms 
within 1-4 days accompanied by normalization of apparent diffusion coefficient (ADC) abnormalities seen on MRI,[9] while cytarabine encephalopathy results in complete resolution in only $40 \%$ of patients because of permanent neurologic deficits.[8] All three patients in this series recovered completely.

\section{Diagnosis}

Diagnosis of LEP consists of history, imaging and histopathology. All patients presented with cerebral findings that pertain to white matter involvement. They also had typical MRI findings of acute chemotherapy-related LEP that included areas of hyperintensity in the white matter involving the periventricular areas, corona radiata and centrum semiovale on T2-weighted images. [9]

On conventional MRI, toxic LEP commonly appears as diffuse T2 and FLAIR images as hyperintense signal in deep periventricular white matter and corpus callosum with sparing of basal ganglia, thalamus and subcortical fibers. Post gadolinium images may or may not show any abnormal contrast enhancement. On diffusion-weighted images (DWI), focal or diffuse areas of reversible restricted diffusion with low ADC can be observed in acute cases, which may show improvement over that period of time if the drug is stopped. However, the corresponding changes on conventional MRI such as T2 and FLAIR hyperintensities may show a larger transitional period for improvement.[10] In 2004, Osborn described the presence of LEP on MRI as periventricular white matter hyperintense edema with diffuse, symmetric involvement of the central and periventricular white matter with relative sparing of the subcortical fibers on T2- weighted images post chemotherapy and radiotherapy. [1 1]

\section{Mechanism of Action and Toxicity}

MTX is a cell cycle-specific agent that inhibits the enzyme dihydrofolate reductase, preventing the conversion of folic acid to tetrahydrofolic acid and inhibiting cell replication. It is one of the most commonly implicated drug causing LEP. [3] The proposed mechanism of MTX-induced LEP is due to accumulation of adenosine, homocysteine elevation and its excitatory effect on the n-methyl-D aspartate (NMDA) receptor. MTX induces disturbances of myelin metabolism or inhibition of glucose metabolism which results in diminished choline to creatine ratio. [1] Choline is an essential nutrient that is required for normal brain development. Its metabolite betaine participates in the synthesis of S-adenosylmethionine, a major methyl donor for histone and DNA methylation, two epigenetic mechanisms that regulate gene expression and may alter brain function. MTX and its metabolite, 7-hydroxymethotrexate, are first taken up by cells where they undergo polyglutamation. Polyglutammated methotrexate (MTXGlu) is an inhibitor of folate-dependent enzymes, such as 5-aminoimidazole-4-carboxamide ribonucleotide (AICAR) transformylase, which will lead to intracellular accumulation of AICAR, a ribonucleotide. This ribonucleotide inhibits AMP deaminase (AMPDA) that will lead to the release of intracellular adenine nucleotides and adenosine, either or both, into the extracellular space. Adenosine dilates cerebral vessels, alters neurotransmitter release and slows neuronal discharge rate.[12] MTX affects homocysteine (Hcy) metabolism which is explained by a lack of the 5-methyltetrahydrofolate required for salvage of $\mathrm{Hcy}$ to methionine. The remethylation of Hcy into methionine is mediated by methionine synthase and its cofactor vitamin B12. Hcy remethylation is an important source of methyl groups in the brain.[13]

Cytarabine is a pyrimidine analog that is incorporated into the DNA during DNA synthesis forcing premature termination of DNA elongation and, ultimately resulting in DNA strand breaks. [3] Cytarabine, a frequent drug used in leukemia, rapidly distributes and crosses the blood-brain barrier and is metabolized by cytidine deaminase which is absent in the central nervous system, providing a theoretical reason for neurotoxicity. [8] Pathologic studies show that cytarabine induces apoptotic death of cultured neurons and loss of Purkinje cells within the cerebellum. Cytarabine disrupts the balance of cyclin-dependent kinases resulting in cell death, induces the formation of reactive oxygen species which cause DNA strand breaks, and ultimately, 
p53-dependent apoptosis. Astrocytes also release soluble factors that increase the susceptibility of neurons to glutamate.[3]

Based on the incidence, clinical presentation and MRI findings, the chemotherapy-induced LEP in these three patients was more likely due to MTX than cytarabine.

\section{Treatment}

No treatment has been proven to prevent or correct neurotoxicity. Most therapeutic reports are anecdotal and treatment effect is difficult to separate from spontaneous resolution of symptom. Aminophylline, an adenosine antagonist, was reported to reverse neurologic toxicity. Dextromethorphan, an NMDA antagonist, has also been reported to reverse MTXassociated neurotoxicity.[4] Some groups had avoided subtle neurotoxicity by giving higher doses of folinic acid. Others suggested giving folinic acid earlier at 36 hours into chemotherapy.[14] Since the occurrence of severe cerebellar dysfunction is greatly affected by age, reduced doses of high-dose cytarabine should be given to patients greater than 50 years old. Very high doses should also be avoided, especially in patients with renal impairment.[15]

Our patients were only placed on supportive measures with adequate hydration without providing any special intervention. Yet, all of them had complete neurological recovery.

\section{CONCLUSION}

Chemotherapy-induced LEP can resolve spontaneously. It may result from direct toxic effects on the nervous system or indirectly from druginduced metabolic derangements or cerebrovascular disorders. Neurotoxicity is most frequently seen with the use of MTX, especially with the IT route. There is no standard treatment for acute and subacute toxicities from MTX. 


\section{REFERENCES}

1. Salkade P, Lim T. Methotrexate-induced acute toxic leukoencephalopathy. J Can Res Ther [Internet]. 2012;8(2):292. Available from: http://dx.doi.org/10.4103/0973-1482.98993

2. Pizzo PA, Poplack DG. Principles and practice of pediatric oncology. 7th ed. Philadelphia, PA: Lippincott Williams and Wilkins; 2016. Chapter 44, p 1113.

3. Cavaliere R, Schiff D. Neurologic toxicities of cancer therapies. Curr Neurol Neurosci Rep [Internet]. 2006 May;6(3):218-26. Available from: http://dx.doi. org/10.1007/s $11910-006-0009-8$

4. Filley CM, Kleinschmidt-DeMasters BK. Toxic Leukoencephalopathy. N Engl J Med [Internet]. 2001 Aug 9;345(6):42532. Available from: http://dx.doi.org/10.1056/ NEJM200108093450606

5. Ziereisen F, Dan B, Azzi N, Ferster A, Damry N, Christophe C. Reversible acute methotrexate leukoencephalopathy: atypical brain MR imaging features. Pediatr Radiol [Internet]. 2005 Dec $21 ; 36(3): 205-12$. Available from: http:// dx.doi.org/10.1007/s00247-005-0015-z

6. Baker WJ, Royer GL Jr, Weiss RB. Cytarabine and neurologic toxicity. JCO [Internet]. 1991 Apr;9(4):679-93. Available from: http://dx.doi.org/10.1200/JCO.1991.9.4.679

7. Vagace JM, de la Maya MD, Caceres-Marzal C, Gonzalez de Murillo S, Gervasini G. Central nervous system chemotoxicity during treatment of pediatric acute lymphoblastic leukemia/lymphoma. Critical Reviews in Oncology/Hematology [Internet]. $2012 \mathrm{Nov} ; 84(2): 274-86$. Available from: http://dx.doi.org/10.1016/i.critrevonc.2012.04.003

8. Peddi PF, Peddi S, Santos ES, Morgensztern D. Central nervous system toxicities of chemotherapeutic agents. Expert Review of Anticancer Therapy [Internet]. 2014 Apr $21 ; 14(7): 857-63$. Available from: http://dx.doi.org/10. 1586/14737140.2014.911089

9. El-Hakam LM, Ramocki MB, Riviello JJ, Illner A. Hyperperfusion on magnetic resonance imaging in acute chemotherapy-related leukoencephalopathy. J Child Neurol [Internet]. 2010 Apr 2;25(6):776-9. Available from: http://dx.doi. org/10.1177/0883073809346349
10. Sindhwani G. MRI in chemotherapy induced leukoencephalopathy: report of two cases and radiologist's perspective. JCDR [Internet]. 2017; Available from: http://dx.doi. org/10.7860/JCDR/2017/29164.10248

11. Osborn AG, Blaser SI, Salzman KL. Diagnostic Imaging: Brain. Canada: AMIRSYS; 2004. Chapter Volume 1 Section 10-46

12. Tian $\mathrm{H}$, Cronstein $B N$. Understanding the mechanisms of action of methotrexate: implications for the treatment of rheumatoid arthritis. Bull NYU Hosp Jt Dis. 2007;65(3): 168-73.

13. Whitehead, M. MD, POG 9903 Polymorphism in methylenetetrahydrofolate reductase (MTHFR) and methotrexate (MTX) neurotoxicity in childhood B-progenitor cell ALL, Dec 2000, p4.

14. Cohen IJ. Neurotoxicity after high-dose methotrexate (MTX) is adequately explained by insufficient folinic acid rescue. Cancer Chemother Pharmacol [Internet]. 2017 Apr 28;79(6):1057-65. Available from: http://dx.doi. org/10.1007/s00280-017-3304-y

15. Smith GA, Damon LE, Rugo HS, Ries CA, Linker CA. Highdose cytarabine dose modification reduces the incidence of neurotoxicity in patients with renal insufficiency. JCO [Internet]. 1997 Feb; 15(2):833-9. Available from: http:// dx.doi.org/10.1200/JCO.1997.15.2.833

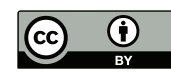

Open Access This article is licensed under a Creative Commons Attribution 4.0 International License, which permits use, sharing, adaptation, distribution and reproduction in any medium or format, as long as you give appropriate credit to the original author(s) and the source, provide a link to the Creative Commons license, and indicate if changes were made. The images or other third party material in this article are included in the article's Creative Commons license, unless indicated otherwise in a credit line to the material. If material is not included in the article's Creative Commons license and your intended use is not permitted by statutory regulation or exceeds the permitted use, you will need to obtain permission directly from the copyright holder. To view a copy of this license, visit http://creativecommons.org/licenses/by/4.0/. 